\title{
ANTS 2012 special issue
}

\section{Editorial}

\section{Marco Dorigo • Mauro Birattari • Christian Blum • Anders Lyhne Christensen • Andries Engelbrecht • Roderich Groß • Thomas Stuitzle}

Received: 7 June 2013 / Accepted: 10 June 2013 / Published online: 26 June 2013

(C) Springer Science+Business Media New York 2013

This special issue of the Swarm Intelligence journal is dedicated to the publication of extended versions of the best papers presented at ANTS 2012, Eighth International Conference on Swarm Intelligence, which took place in Brussels on September 12-14, 2012.

M. Dorigo $(\bowtie) \cdot$ M. Birattari · T. Stützle

IRIDIA, Université Libre de Bruxelles, Brussels, Belgium

e-mail: mdorigo@ulb.ac.be

\section{Birattari}

e-mail: mbiro@ulb.ac.be

T. Stützle

e-mail: stuetzle@ulb.ac.be

C. Blum

Department of Computer Science and Artificial Intelligence, University of the Basque Country, San Sebastian, Spain

e-mail: christian.blum@ehu.es

C. Blum

IKERBASQUE, Basque Foundation for Science, Bilbao, Spain

\section{A.L. Christensen}

Instituto Universitário de Lisboa (ISCTE-IUL) \& Instituto de Telecomunicações, Lisbon, Portugal e-mail: anders.christensen@iscte.pt

\footnotetext{
A. Engelbrecht

CIRG, Department of Computer Science, University of Pretoria, Pretoria, South Africa e-mail: engel@cs.up.ac.za

R. Groß

Natural Robotics Lab, Department of Automatic Control and Systems Engineering, The University of Sheffield, Sheffield, UK

e-mail: r.gross@sheffield.ac.uk
} 
The ANTS series of conferences has taken place at the Universite Libre de Bruxelles, Brussels, Belgium, every other year since 1998. As in 2010 (for the seventh edition of the conference), the authors of the contributions accepted as full papers at the conference were invited to submit an extended version of their work for possible inclusion in this special issue.

Six of the submitted papers were accepted for publication after at least one round of reviews with comments by at least three referees. The review process for papers where one of the authors was also one of the editors of this special issue has been managed anonymously by one of the other guest editors.

The special issue opens with "Modular self-assembling and self-reconfiguring e-pucks" by Murray, Timmis and Tyrrell. In this paper, the authors present a passive, low-cost structural extension for the e-puck robotics platform that allows multiple robots to self-assemble into a single physical structure. This extension effectively transforms a widely used mobile robotics platform into a self-reconfigurable modular robotics platform. The authors develop a collective locomotion algorithm that enables connected robots to move and avoid obstacles as a single entity. The algorithm and the structural extension are tested extensively in a series of real robot experiments with up to nine connected robots. The authors also demonstrate self-assembly and self-disassembly triggered by an environmental cue. Notably, the designs for the structural extension are freely available online.

In "Evolution of swarm robotics systems with novelty search", Gomes, Urbano and Christensen explore evolutionary algorithms for the design of control systems for swarms of robots. In particular, algorithms based on Lehman and Stanley's novelty search are considered, where the fitness depends on the behavioural novelty of proposed solutions rather than on a task specific objective function. Two case studies of different complexity are considered that require a swarm of mobile robots to aggregate and to share a resource- a charging station. The results show that the approach works well and is unaffected by premature convergence. As such, novelty search is particularly suited to scenarios in which traditional objective function designs fail due to deceptive fitness landscapes. In addition, the authors study an extension to the approach, which combines the explorative power of novelty search with the exploitative power of traditional objective function based approaches.

In "Towards swarm calculus: urn models of collective decisions and universal properties of swarm performance", Hamann presents and analyses two models related to swarm intelligence. This is a first step in the quest for a 'swarm calculus', which should ultimately allow researchers to derive key features of artificial and natural swarms, such as performance and robustness. The first of these models describes the performance of a swarm as a function of the swarm density; in this context, particular attention is given to the dichotomy between cooperation and interference. The second model is inspired by urn models and addresses collective decision making. Applications of the models to both artificial and natural swarms are also described.

In "Task partitioning in a robot swarm: a study on the effect of communication", Pini et al. investigate whether it is beneficial for a swarm of robots to partition an overall task into sub-tasks to be tackled separately. The problem is formulated as a multi-armed bandit, where each robot chooses whether to work on the whole task or on the sub-tasks. A foraging scenario is considered. The performance of an ad-hoc algorithm is compared against stateof-the-art algorithms from the multi-armed bandit literature. Particular emphasis is given to the role of explicit communication between the robots. It is found that although communication can speed up the decision making process, it may also make the swarm less adaptive to dynamic environments.

In "On the use of Bio-PEPA for modelling and analysing collective behaviours in swarm robotics", Massink et al. present a novel and potentially powerful approach for modelling 
and analysing swarm robotics systems. The approach is based on Ciocchetta and Hillston's Bio-PEPA, which was originally developed in the context of biochemical networks. BioPEPA is a timed process algebra, which integrates a wide range of modelling and analysis techniques, starting from a single microscopic specification. After introducing Bio-PEPA, the paper presents a case study in which a swarm of robots is required to choose collectively the shortest of two paths in a foraging scenario. The case study illustrates how the Bio-PEPA suite facilitates modelling and analysis via stochastic simulation, numerical integration of ordinary differential equations, and model checking.

In "Learning Bayesian network classifiers using ant colony optimization", Salama and Freitas apply ACO algorithms to a specific machine learning task, namely the generation of classifiers. They develop the ABC-Miner algorithm that optimises the structure of a Bayesian network classifier, which is a specific type of Bayesian network that is used for classification. The experimental evaluation of ABC-Miner shows that it improves upon wellknown algorithms for constructing Bayesian network classifiers and that it obtains classification performance competitive to state-of-the-art methods.

The six papers contained in this special issue are of very high quality. We thank all the people who made this possible: the authors, who submitted their best work to the journal; the referees, who helped in the selection of these six papers out of the 81 submitted to ANTS 2012; and finally, the many people at Springer who assisted us in the production of this special issue.

June 2013

\author{
Marco Dorigo \\ Mauro Birattari \\ Christian Blum \\ Anders Lyhne Christensen \\ Andries Engelbrecht \\ Roderich Groß \\ Thomas Stützle
}

